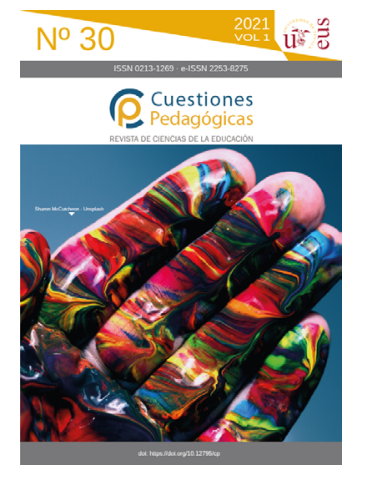

\title{
Estrategias de enseñanza para niños, niñas y adolescentes jornaleros agrícolas migrantes en México
}

\author{
Teaching strategies for migrant farm children and \\ adolescents in México
}

Recibido: 13/11/2020 | Revisado: 05/05/2021 | Aceptado: 08/06/2021 |

Online first: 10/06/2021 | Publicado: 01/07/2021

D Diego Juárez-Bolaños

Universidad Iberoamericana Ciudad de México (México)

diego.juarez@ibero.mx

https://orcid.org/0000-0003-4221-0885

\section{Luis Ernesto Solano-Becerril}

Universidad de La Salle Bajío (México)

Isolano@delasalle.edu.mx

https://orcid.org/0000-0001-5903-5433

\section{Juana Isabel Cervantes-Gómez}

Universidad de La Salle Bajío (México)

isacervantes675@gmail.com

https://orcid.org/0000-0002-1064-7110

\section{Luz Elena Vega-Santos}

Universidad de La Salle Bajío (México)

lu_evs@hotmail.com

https://orcid.org/0000-0002-5998-0723

\begin{abstract}
Resumen: Las familias que habitan de los territorios rurales desarrollan diversas estrategias para obtener ingresos económicos, tales como el trabajo en la propia tierra, la venta de artesanías, la migración nacional e internacional. En este sentido, existen miles de familias en México que realizan procesos temporales de migración interna, que viajan a campos agrícolas nacionales para trabajar en la cosecha de diversos productos durante algunos periodos de tiempo. Buena parte de estas familias migrantes son acompañadas por niños y adolescentes en edad escolar. La literatura sobre la educación formal de los niños, niñas y adolescentes jornaleros agrícolas migrantes (NNAJAM) se ha centrado en aspectos contextuales, en las acciones y programas de gobierno y en las condiciones escolares, pero poco se ha escrito sobre las estrategias de enseñanza en las aulas. El presente artículo tiene como objetivo sistematizar y examinar diversas estrategias de enseñanza que han sido aplicadas en escuelas que atienden a estas poblaciones en México. Los datos provienen de una investigación documental, a través de la cual identificó y sistematizó la literatura que, de manera central o secundaria, ha mencionado,
\end{abstract}

Abstract: The families that live in rural territories develop various strategies to obtain economic income, such as working on their own land, selling handicrafts, and national and international migration. In this sense, there are thousands of families in Mexico who carry out temporary internal migration processes, who travel to national agricultural fields to work in the harvest of various products for some periods of time. A good part of these migrant families are accompanied by children and adolescents of school age. The literature on the formal education of migrant agricultural day laborers (NNAJAM, acronym in Spanish) has focused on contextual aspects, government actions and programs and school conditions, but little has been written about teaching strategies in the classrooms. The objective of this article is to systematize and examine various teaching strategies that have been applied in schools that serve these populations in Mexico. The data come from a documentary research, through which he identified and systematized the literature that, in a central or secondary way, has mentioned, described or examined the teaching strategies for children and adolescents. The main contributions of the arti- 
descrito o examinado las estrategias de enseñanza para los NNAJAM. Los principales aportes del documento se centran en reunir, sistematizar y analizar diversas estrategias implementadas en escuelas en las áreas de lenguaje, razonamiento matemático, aprendizaje colaborativo, interculturalidad, uso del tiempo libre y alimentación y nutrición.

Palabras clave: educación migrante, docencia, experiencia en la enseñanza, educación rural, educación para agricultores. cle focus on gathering and analyzing strategies in the areas of Language, Mathematica, Collaborative Learning, Interculturality, Use of Free Time and Nutrition.

\section{Introducción}

En varias naciones existe una población que vive en condiciones sociales precarias, pero su participación en la vida social es vital pues atienden las cadenas alimentarias, nos referimos a las familias jornaleras agrícolas migrantes ${ }^{1}$ : personas que migran de manera interna en sus propios países, desde sus comunidades de origen hacia campos agrícolas ubicados en diversas regiones integradas a cadenas agroproductivas, muchas de éstas relacionas con productos de exportación.

En el caso de México, estos procesos migratorios tienen algunas características distintivas. Generalmente, se realizan de manera grupal-familiar puesto que las comunidades de origen ofrecen una limitada dotación de servicios públicos tanto en cantidad como en calidad, además de la falta de oportunidades productivas y laborales, así como salarios precarios; y los procesos migratorios suelen ser por temporadas agrícolas, volviendo los migrantes internos a sus localidades de origen cada cierto tiempo.

En el país, no se sabe exactamente cuántos son: la más reciente estimación se realizó en el año 2009 (SEDESOL, 2011) y se calculaba que habían poco más de 2 millones de personas en esa condición, de los cuales, la Secretaría de Educación Pública (SEP) calculaba que existían entre 279 y 326 mil niños, niñas y adolescentes agrícolas migrantes (NNAJAM) en edad de cursar la educación básica (INEE, 2016).

Tal como señaló el Instituto Nacional de Evaluación de la Educación (2016, p. 7) esta población sufre diversas carencias educativas:

(...) la falta de disponibilidad de servicios educativos o de oportunidades para una trayectoria escolar continua, la atención por parte de figuras educativas con formación o experiencia profesional insuficiente y la carencia de mobiliario y condiciones adecuadas para el aprendizaje en sus escuelas (...) se estima que sólo entre 14 y $17 \%$ de NNA de familias de jornaleros agrícolas migrantes asiste a la escuela, lo que sin duda refleja las limitaciones que enfrentan para acceder a la educación.

Aunado a estas condiciones, los estudios Ramírez (2000), Salinas (2004), INEE ( 2016) y Glockner (2019) entre otros, documentan las situaciones de vulnerabilidad y desigualdad en que las familias migrantes jornaleras agrícolas viven, y en específico, la niñez que los acompaña, pues constantemente participan en el mercado de trabajo como un adulto más de la unidad familiar. Se esta manera, se vulneran los derechos de los niños, ya que el trabajo infantil está prohibido en México (Artículo 123 constitucional) y se limitan sus derechos para recibir educación inclusiva, de calidad, pertinente y contextualizada, excluyéndolos de las relaciones sociales y afectivas que le permitan un desarrollo acorde con sus necesidades y aspiraciones (Vera, 2007).

1 Así se les conoce en México a este grupo poblacional, pero no ocurre así en toda América Latina, por ejemplo, en Brasil se denominan Trabajadores Rurales en Movimiento; en Argentina, Trabajadores Transitorios Agropecuarios; en otros países como Familias Campesinas Migrantes o Familias Asalariadas del Campo, dependiendo de las características socio-productivas de las unidades domésticas. La Organización de las Naciones Unidas para la Alimentación y la Agricultura (FAO) las ubica dentro del grupo general de trabajadores rurales. 
A lo anterior, se suma la existencia de contextos sociales donde confluyen condiciones materiales y educativas desafiantes, propias de una población sujeta a complejas dinámicas de movilidad, explotación social y económica, precarización de la vida y el trabajo, así como una gran heterogeneidad lingüística y cultural (Rojas en Glockner, 2019).

La literatura sobre la educación de los NNAJAM se ha centrado en los aspectos contextuales (condiciones de vida y de trabajo en las comunidades de origen y destino), en las acciones y programas de gobierno que se han desarrollado (o no) para atender educativamente a esta población y en las condiciones escolares (formación y características de las figuras educativas, infraestructura y equipamiento de los centros escolares, materiales educativos). Sin embargo, son limitadas las investigaciones en México que se han adentrado de manera específica en las estrategias de enseñanza dirigidas a esta población. Es por ello, que el presente artículo tiene el objetivo de sistematizar y analizar parte de la literatura que, de manera central o secundaria, ha abordado las estrategias de enseñanza propuestas o desarrolladas para los NNAJAM².

\section{Recuento de acciones y programas educativos dirigidos a los NNAJAM}

El Estado mexicano ha desarrollado algunos programas y acciones que han pretendido brindar el servicio educativo a NNAJAM. En la búsqueda de datos localizamos 14 documentos (tres tesis, tres evaluaciones, una ponencia en congreso, tres materiales educativos y cuatro de artículos de revistas) que mencionan de manera central y secundaria este tema.

Las figuras educativas que laboran en las escuelas rurales y multigrado y las que atienden a población jornalera migrante tienen similitudes, las cuales están presentes en el contexto y la organización de atender a estudiantes de diferentes grados en un mismo grupo y pueden o no tener heterogeneidad cultural, pero las diferencias radican en que las escuelas para NNAJAM están a expensas de conseguir espacios físicos, la contratación docente depende de la cantidad de estudiantes que lleguen a comunidad y de su diversidad étnica.

Además, los docentes deben realizar constantes adecuaciones al currículum nacional a fin de contextualizarlo a las características de los grupos de estudiantes que atienden (INEE, 2018; Galván y Espinosa, 2107; Arteaga, 2009). Lo anterior, porque el currículum ha sido pensado y diseñado para escuelas preescolares y primarias unigrado (es decir, en donde un docente atiende a un grupo) localizadas preferentemente en zonas urbanas y en poblaciones con un mayor número de habitantes. En las escuelas multigrado, donde los maestros atienden a más de un grupo a la vez, es complejo abordar todos y cada uno de los contenidos plasmados en los planes y programas de estudio para cada grado, por lo cual se deben elegir qué temas son los centrales en relación al contexto sociocultural del alumnado.

Entre los años 2002 y 2014 se llevaron a cabo algunas acciones de atención educativa a la población jornalera migrante como la Modalidad Educativa Intercultural para Población Infantil Migrante (MEIPIM) desarrollada por el Consejo Nacional de Fomento Educativo (CONAFE) o el Programa de Educación Primaria para Niñas y Niños Migrantes (PRONIM) (como componente del Programa de Inclusión y Equidad Educativa-PIEE) por parte de la Secretaría de Educación Pública (SEP), con el objetivo de ampliar y mejorar la oferta educativa para la población infantil migrante.

De 1990 a 2018 operó el Programa de Apoyo a Trabajadores Jornaleros Agrícolas (PAJA) a cargo de la Secretaría de Desarrollo Social (SEDESOL), hoy Secretaría del Bienestar. En el Presupuesto de Egresos de la Federación (PEF) del año 2019 tal programa desapareció, sin ser sustituido por algún otro y sin dar a conocer los estudios técnicos o evaluaciones de impacto que justificasen tal acción. El PAJA tenía un componente educativo, ya que otorgaba becas a los NNAJAM para estimular su

2 El presente documento se deriva del proyecto "Metodologías de Enseñanza-Aprendizaje para niñas, niños y adolescentes de familias de jornaleros migrantes", financiado por la Secretaría de Educación de Guanajuato, el cual forma parte del banco de proyectos del Sistema de Redes de Investigación Educativa Aplicada. 
asistencia y permanencia en las escuelas ${ }^{3}$. El monto de los apoyos dependía del grado escolar y sexo del alumnado (SEDESOL, s/f).

Entre los años 2003 al 2006 existió el proyecto Fomentar y Mejorar la Educación Intercultural para los Migrantes (FOMEIM). Fue un proyecto de coordinación interinstitucional e intersectorial en el que participaron la Coordinación General de Educación Intercultural y Bilingüe (CGEIB), el CONAFE, el Instituto Nacional para la Educación de los Adultos (INEA), la Dirección General de Educación Indígena (DGEI), la Dirección General de Desarrollo de la Gestión e Innovación Educativa (DGDGIE) y el Programa Infancia de la Universidad Autónoma Metropolitana (UAM) (Chapela, 2006). Además, algunas entidades como Baja California y Baja California Sur elaboraron algunos materiales específicos para escuelas de NNAJAM. No localizamos evaluaciones sobre el impacto del FOMEIN y existe poca evidencia de sus resultados entre las que destaca el aporte de Salinas (2004).

La Modalidad Educativa Intercultural para Población Infantil Migrante (MEIPIM) fue implementada en los centros escolares atenidos por el Consejo Nacional de Fomento Educativo (CONAFE) en 1997. Con la puesta en marcha de un nuevo modelo educativo llamado Aprendizaje Basado en la Colaboración y el Diálogo, se eliminó el MEIPIM, desapareciendo así la atención educativa diferenciada y específica para poblaciones migrantes que ofrecía CONAFE (Juárez y Lara, 2018). Entre las características de la MEIPIM se incluían el trabajo a partir de proyectos educativos planteados por los niños y con actividades relativas a un problema relacionado con la vida de las comunidades migrantes, además del fomento de,

(...) aprendizajes significativos a partir de la experiencia migratoria agrícola (...) en las actividades y estrategias didácticas, las cuales deberían corresponder al contexto de la migración, (...) propone que la niñez migrante avance en el conocimiento a través del planteamiento y la resolución de problemas reales a los que se enfrenta tanto en su vida diaria, como en las labores que realiza (Pacheco et al., 2016, p.95).

Los ejes curriculares de la MEIPIM fueron comunicación, razonamiento lógico-matemático, comprensión del medio natural, social y cultural, actitudes y valores para la convivencia y aprender a aprender (Garduño, 2000). El currículo procuraba centrarse en la problematización de las prácticas y los saberes culturales del niño migrante para la satisfacción de sus necesidades a través de dos ejes: Plantear y Resolver Problemas y el Juego.

El primero procuraba desarrollar en los estudiantes "formas de razonamiento y acción útiles para abordar cualquier problema dentro o fuera de la escuela", mientras que el segundo eje reconocía que "como todos los niños y niñas, tienen derecho a jugar y a tener espacios de juego, enfrentarse y resolver este problema, constituye una de sus necesidades básicas, que la escuela puede contribuir a resolver, ofreciéndoles la oportunidad de analizar, discutir, planear y decidir, qué juguetes construir y qué espacios utilizar" (Garduño, 2000, pp. 15 y 16). Esta modalidad educativa funcionó hasta 2016.

Tanto la SEP como CONAFE intentaron desarrollar durante más de una década programas que atendieran a los NNAJAM con la especificidad que requieren, sin embargo, en los últimos años han ido desapareciendo. La Modalidad Educativa Intercultural para Población Infantil Migrante del CONAFE (Garduño, 2000) trató de contemplar las características socioculturales de la población estudiantil migrante. Sin embargo, no localizamos estudios que dieran cuenta sobre los resultados de su implementación, y tal acción terminó ya que el tiempo y el financiamiento con el que estaba implementado (Fondo Mixto de Cooperación Técnica y Científica México- España y la SEP) cumplió el tiempo acordado (Rojas, 2011). Lo mismo ocurrió con el proyecto Fomentar y Mejorar la Educación Intercultural para los Migrantes (FOMEIM) finalizado en 2006 y con el Programa de Apoyo a Trabajadores Jornaleros Agrícolas (PAJA) terminado en 2019.

3 Aunque a lo largo del artículo hablamos de escuelas o centros educativos, debemos reconocer que la atención a los NNAJAM se realiza a través de diversos espacios con diversas denominaciones dependiendo de qué autoridad lo sostenga. De esta manera, existen Centros de Atención Integral para Niños Migrantes (CAINMI), Centros de Educación Preescolar Indígena (CEPI), centros escolares ubicados en campamentos migrantes, entre otros. 
Lo anterior nos muestra un tema recurrente en las políticas sociales: la desaparición de programas por parte de autoridades de diferentes niveles de gobierno, sin sustentos ni argumentos y debilitando la ya de por sí precaria atención educativa a poblaciones vulnerables, como los NNAJAM.

Uno de los componentes del PRONIM fue elaborar una propuesta curricular organizada en módulos relacionados con los ciclos agrícolas, por lo que se diseñaron cinco módulos correspondientes a cada uno de los meses de trabajo escolar (Ramírez, 2008). La propuesta se apoyaba en los siguientes materiales:

, Guía para el docente. Español y el medio natural y social. $1^{\circ}, 2^{\circ}$ y $3 e r$ ciclo ${ }^{4}$.

> Guía para el docente. Matemáticas. $1^{\circ}, 2^{\circ}$ y $3 e r$ ciclo.

, Fichero de actividades para la educación primaria de niñas y niños migrantes.

, Español y el medio natural y social. $2^{\circ}$ y $3 e r$ ciclo.

> Fichero de actividades para la educación primaria de niñas y niños migrantes.

, Matemáticas. $2^{\circ}$ y $3 e r$ ciclo.

Estos materiales orientaban al docente para trabajar con grupos multigrado, al mismo tiempo que intentaban promover el trabajo autónomo de los alumnos en los ciclos segundo y tercero, mientras se atendían las necesidades de la adquisición de la lectoescritura en el primer ciclo (SEP, 2006). El tiempo de trabajo académico que se contemplaba en las Guías y en las Ficheros de actividades era de cuatro horas diarias, cinco días a la semana, durante cinco meses, tomando como referencia el promedio de asistencia de los niños al aula, tanto en zonas de origen como de atracción (SEP, 2006).

De acuerdo con la SEP (2006),

La Guías y las Fichas tienen como propósito facilitar al maestro la planeación, organización y realización de su trabajo en un grupo multigrado, considerando las condiciones limitadas que tienen las niñas y los niños migrantes para asistir y permanecer en la escuela durante periodos prolongados. Debido a las dificultades que los niños tienen para cubrir la totalidad de los contenidos del Plan y Programas de Estudio vigente, se hizo una selección de contenidos, dando prioridad a aquellos que les permiten el desarrollo de la lengua oral y escrita, el cuidado de la salud y el medio ambiente, el reconocimiento y respeto a la diversidad cultural, así como al fortalecimiento de su identidad individual y nacional.

La Universidad Pedagógica Nacional (UPN) encabezó evaluaciones externas al PRONIM del 2002 al 2006, una de éstas desarrollada en diversas entidades del país (Rodríguez y Ramírez, 2006) identificó que la propuesta curricular era útil al proporcionar estrategias y herramientas a los docentes que facilitaban el trabajo en las aulas, debido a la simplificación de los contenidos. Sin embargo, se reconocía que para ser desarrollada se requería una sólida formación docente.

En el año 2013 el PRONIM fue integrado, junto con otros programas de la SEP relacionados con atención educativa a alumnos con discapacidad, estudiantes con aptitudes sobresalientes, escuelas indígenas, multigrado y multigrado, al Programa para la Inclusión y la Equidad Educativa (PIEE) (DOF, 2014). Esta unificación debilitó las acciones hacia poblaciones vulneradas en el cumplimiento a sus derechos educativos, ya que al PIEE se le asignó un presupuesto menor a la suma de los programas que lo integraron, además de eliminar puestos de trabajo de equipos técnicos formados en diversas áreas de especialidad educativa. EI propio gobierno federal recomendó a la SEP aclarar los criterios que se tomaron en cuenta para fusionar los siete programas el marco del derecho a una educación de calidad, independientemente de la sola conveniencia de simplificar el proceso presupuestario (SEP, 2019) siendo ignorada esta petición.

Uno de los objetivos del PIEE fue "Beneficiar a centros educativos migrantes, incluyendo el nivel de secundaria, con acciones de fortalecimiento académico, apoyos específicos, contextualización de contenidos; así como equipamiento específico y diversificación curricular" (SEP, 2019, p. 11).

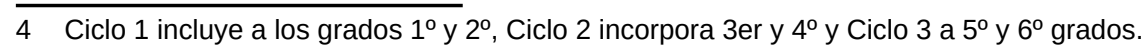


Comparando los materiales elaborados por la SEP (PROMIN) y CONAFE encontramos coincidencias, ambos modelos intentaban relacionar los saberes familiares con los conocimientos científicos disciplinares, promovían el trabajo colaborativo entre estudiantes de diversos ciclos, grados, experiencias, intereses y edades, además de adaptarse a las circunstancias de los alumnos que requieren pedagogías flexibles debido a la asistencia intermitente, su trabajo asalariado o en las tareas domésticas y la alta rotación de alumnos en las escuelas a lo largo del ciclo escolar gracias a la dinámica agrícola. Rojas (2006, p. 117) establecía que a tres años de haberse implementado el PRONIM, aún mostraba pocos resultados en el ámbito pedagógico:

Uno de los grandes retos pedagógicos y didácticos que aún no se ha resuelto es que los maestros conozcan cómo enseñarles a leer y escribir, la enseñanza y uso de la primera y segunda lengua en las actividades escolares; atención a la diversidad cultural y lingüística que se les presenta en los salones de clase; el desarrollo de actividades educativas en los grupos multigrados con los que laboran; entre otros aspectos necesarios que desconocen para poder lograr aprendizajes significativos en el primer grado.

Lo anterior debido a múltiples factores, entre los que Rojas (2006) identifica la falta de infraestructura y aulas dignas, precarización de las condiciones laborales de los docentes y asesores, formación adecuada y pertinente de los maestros y falta de permanencia y retención del alumnado. A casi tres lustros de tales señalamientos, parecería que la situación no ha mejorado, incluso se ha empeorado con la desaparición del PRONIM, uno de los pocos programas educativos federales diseñados para los NNAJAM.

Una vez realizada esta revisión contextual de las acciones y políticas desarrolladas por diversas instancias gubernamentales, nos abocaremos a detallar las estrategias específicas de enseñanza que se han utilizado particularmente para los NNAJAM, lo cual puede ser de interés para las figuras educativas que trabajan con estas poblaciones.

A esta suma de métodos, técnicas, actividades, acciones que el docente utiliza para alcanzar sus propósitos se les llaman estrategias didácticas pues son los medios y los recursos que se ajustan para lograr aprendizajes a partir de la intencionalidad del proceso educativo (Díaz Barriga y Hernández Rojas, 2010: 118). Ferreiro (2005), coincide cuando define a las estrategias de enseñanza como procedimientos empleados por el docente para hacer posible el aprendizaje del estudiante y que incluyen operaciones físicas, mentales y otros elementos para facilitar la confrontación del sujeto que aprende con el objeto de conocimiento, es decir, las acciones que realiza el docente para enseñar al educando. Las estrategias de enseñanza que encontramos en nuestra investigación documental se detallan a continuación.

\section{Estrategias didácticas para la enseñanza de la lectoescritura}

En este apartado incluimos la revisión de documentos con contenido significativo para incentivar los procesos de la enseñanza de la lectoescritura para NNAJAM (Schmelkes, 2002; Rojas, 2011; Ávila, 2013; Rubio, 2009; Feltes y Reese, 2014; Blancas, 2017).

La lectura y la escritura son elementos esenciales para el proceso de comunicación interpersonal y para el proceso de enseñanza aprendizaje. A través del lenguaje hablado se expresa el sentir, las necesidades y la cultura, mientras que la lengua escrita es una forma de expresión del lenguaje que implica una comunicación simbólica con ayuda de signos elegidos por la persona y en tal sentido arbitrados y convencionales (Blancas, 2017). De igual forma la lectura permite comprender las diferentes realidades e interactuar con la información que está inserta en un libro, en una revista, en un anuncio, en el pizarrón, en una libreta, y esa interacción existe con lo que está escrito y con la información que se presenta de manera visual, como, las imágenes o dibujos. Es conveniente que la enseñanza de la lecto-escritura se apoye en las costumbres, valores y habilidades que las comunidades y las familias poseen (Montoya y Villarreal, s.f).

Ahora bien, varios de los NNAJAM presentan dificultades para aprender a leer y a escribir debido a la falta de atención a la diversidad lingüística. Según el INEE (2019, pág. 12-13) para 2018 había 
5,996,318 personas indígenas sólo en el ámbito rural y de los cuales 4,328,295 oscilan entre los 0 a 17 años, estos niños y niñas tuvieron como lengua materna la lengua indígena, sus primeros años de vida, por lo que es evidente que sean más competentes en dicha lengua (Ávila, 2013) lo que presenta un reto para el Estado: adecuar estrategias de enseñanza - aprendizaje en contextos de diversidad lingüística y para entornos de migración, capacitación docente y contratación, entre otras problemáticas correlacionadas. En un estudio que se realiza con los docentes dentro de los espacios educativos para NNAJAM se puede percibir la falta de capacitación para la enseñanza en lenguas originarias (Ávila, 2013) ${ }^{5}$. Por lo que, al ingresar un estudiante hablante de una lengua originaria a estos espacios educativos, la diferencia de idioma se convierte en una barrera que limitará su aprendizaje.

En la revisión de la literatura documental se encontraron ciertas estrategias didácticas, actividades y uso de recursos utilizados para la enseñanza, aprendizaje o fortalecimiento en esta área, estas estrategias se eligieron debido a que existe evidencia de su aplicación en los campos agrícolas.

Las estrategias se sintetizan en la Tabla siguiente.

\section{Tabla 1}

Estrategias de enseñanza para fortalecer el lenguaje y lectoescritura

\section{Estrategia Descripción de actividades ylo uso de materiales}

El concepto de doble inmersión se refiere a un modelo de educación bilingüe en donde la enseñanza de los contenidos académicos es en dos idiomas y se trabaja por separado.

Aplicar programas de doble inmersión que permitan atender la diversidad lingüística.

\begin{tabular}{l} 
División de con- \\
tenidos por eje \\
lingüístico y de \\
comunicación: \\
hablar, escuchar, \\
leer y escribir. \\
\hline
\end{tabular}

Planear espacios de interacción mediante la expresión oral y reflexión cultural.

Uno de estos modelos es el 50-50 "en el cual cada lengua es utilizada durante cincuenta por ciento del día para la instrucción en todos los niveles de grado" (Howard y Christian, 2002). Esta estrategia estaría limitada o inaplicable en caso de que existan más de dos lenguas en un mismo espacio.

Asimismo, el modelo 90- 10 en la cual la instrucción en la lengua minoritaria se da durante noventa por ciento de cuarto grado en adelante (Howard \& Christian, 2002)".

Para la implementación de esta estrategia dentro del PRONIM, los docentes organizaban las temáticas de aprendizaje en "Expresión oral, Lectura, Escritura y Reflexión sobre la lengua". A partir de ello se organizan los contenidos y las estrategias de enseñanza- aprendizaje.

Realizar actividades como: mesas de diálogo, debates y asambleas, para fortalecer el uso del lenguaje hablado como medio de aprendizaje. Para llevar a cabo las actividades es importante acordar un tema que se vincule con el contexto inmediato de los estudiantes, con situaciones que conozcan (la familia, las fiestas, los juegos), que les emocionan o les gustan, poner atención en su forma de expresión y corregir, fomentar el debate de ideas y acuerdos para llegar a soluciones.

Dentro de los talleres se proponen diversas actividades, utilizando el lenguaje escrito mediante la creación de algunos textos, como escribir men-

Diseñar talleres para el fomento de la escritura

Implementar intervalos de lectura acompañados por actividades previas, durante y después de la lectura. sajes a sus compañeros familiares o vecinos utilizando recados, asimismo, la creación de cuentos, folletos, informes, recetarios, relatos entre otras cosas. Dentro de este taller es importante planear, redactar, revisar y corregir los diferentes tipos de textos.

Para esto es importante contar con un acervo de libros. Para realizar los espacios de lectura y fortalecerla se proponen tres tiempos: antes de leer es importante incluir actividades de preguntas previas al tema que se va a leer, durante la lectura es de suma importancia continuar con preguntas que permitan la reflexión de lo que se está leyendo e imaginen los sucesos que vienen después, al final de la lectura implementar una actividad que tenga como objetivo la comprensión y resignificación.
Documentado por

Howard y Christian citado en Feltes y Reese (2014)

Revisión del PRONIM por Rubio (2009)

5 Ávila (2013) se refiere a segundas lenguas cuando en realidad la dualidad está entre lenguas originarias o indígenas y el español. 


\begin{abstract}
Uso del juego como herramienta de aprendizaje.
\end{abstract}

\begin{tabular}{|c|c|}
\hline $\begin{array}{l}\text { El uso de animales o cosas para el pase de lista, tiene como objetivo que } \\
\text { los estudiantes inicien con el aprendizaje del abecedario "y les sea más } \\
\text { significativa la manera de ir formando su nombre y otras palabras que em- } \\
\text { piecen con la misma letra". Esta actividad consiste en identificar la letra del } \\
\text { abecedario con la que empieza el nombre. Se dibuja un animal o cosa que } \\
\text { esté relacionada a su contexto y se expone. } \\
\text { De igual forma, el juego de "Basta". Tiene como objetivo que el alumno se } \\
\text { apropie de los sonidos de las letras y relacione las palabras con la grafía, } \\
\text { consiste en hacer un cuadro con tres columnas (nombre, fruta y cosa) en } \\
\text { la parte posterior se debe escribir el abecedario, se repetirá el abecedario } \\
\text { en voz alta y cuando se diga la palabra "basta", se escriben los datos en la } \\
\text { tabla de acuerdo con la letra que haya tocado. } \\
\text { Además, es importante trabajar con la memoria, por lo que se recomienda } \\
\text { el juego de "Memorama" que consiste en otorgar las cartas o tarjetas, estas } \\
\text { tarjetas deberán contener figuras, objetos, animales etc., estas se ponen } \\
\text { boca-abajo, por turnos ir destapando las cartas tratando de encontrar el } \\
\text { par, cuando lo hagan escribirán en su cuaderno el nombre de la figura u } \\
\text { objeto fortaleciendo la relación entre imagen, sonido y grafía. }\end{array}$ & $\begin{array}{l}\text { Montoya y Villareal, } \\
\text { (s.f.) }\end{array}$ \\
\hline $\begin{array}{l}\text { Para continuar con el trabajo de los nombres se utilizan materiales como: } \\
\text { semillas, pastas, piedras y una hoja de papel, con estos materiales se } \\
\text { trabaja la construcción de su nombre, para esto es necesario marcar en } \\
\text { grande su nombre en la hoja para que los estudiantes peguen los materi- } \\
\text { ales en el contorno. }\end{array}$ & \\
\hline
\end{tabular}

El uso de animales o cosas para el pase de lista, tiene como objetivo que los estudiantes inicien con el aprendizaje del abecedario "y les sea más significativa la manera de ir formando su nombre y otras palabras que empiecen con la misma letra". Esta actividad consiste en identificar la letra del abecedario con la que empieza el nombre. Se dibuja un animal o cosa que esté relacionada a su contexto y se expone. la parte posterior se debe escribir el abecedario, se repetirá el abecedario en voz alta y cuando se diga la palabra "basta", se escriben los datos en la tabla de acuerdo con la letra que haya tocado.

Además, es importante trabajar con la memoria, por lo que se recomienda el juego de "Memorama" que consiste en otorgar las cartas o tarjetas, estas tarjetas deberán contener figuras, objetos, animales etc., estas se ponen boca-abajo, por turnos ir destapando las cartas tratando de encontrar el par, cuando lo hagan escribirán en su cuaderno el nombre de la figura u objeto fortaleciendo la relación entre imagen, sonido y grafía.

Utilizar recursos del contexto para el desarrollo de actividades. 
comprender la diversidad de procesos de enseñanza aprendizaje que están interactuando dentro del salón de clases y reflexionar sobre la flexibilidad de la escuela para que los alumnos se expresen los saberes no formalizados (Gutiérrez, 2013).

A partir de dibujos se pudieron recuperar aportes valiosos respecto a los conocimientos que los niños tenían en cuanto a las prácticas productivas y los conocimientos locales (cultivo de maíz y la adecuación de utensilios) así como sus experiencias y apreciaciones sobre las condiciones migratorias de sus padres. Por su parte, Vera y Yocupicio (2007) recomiendan que, ante la imposibilidad del docente de conocer 10 idiomas diferentes, el empleo de dibujos, mímica, o demás recursos lúdicos ayudan a establecer un puente entre docentes y estudiantes.

Méndez y Huguette (2013) mencionan que, a lo largo de los traslados inherentes a la migración, los y las NNAJAM van adquiriendo aprendizajes que les permiten solucionar problemas relacionados con la realización de trámites, la visita al médico, al banco o la oficina. Dichas experiencias, aun cuando son valiosas, suelen quedar excluidos en el espacio escolar debido a que no se encuentra una relación con las materias tradicionales.

Finalmente, partiendo del principio de que la cultura y las experiencias de vida son decisivas en la construcción de significados y la asimilación de conocimientos se debe contemplar la adecuación de las prácticas pedagógicas para integrar los aprendizajes no formales que los estudiantes han generado en su vida cotidiana. Con ello se evitará preservar dinámicas exclusivas y se enriquecerán las actividades dentro del aula, propiciando el apropiamiento del proceso de enseñanza-aprendizaje, el encuentro intercultural y el desarrollo de habilidades socioemocionales de los y las NNAJAM.

Tabla 2

Estrategias didácticas para fortalecer el aprendizaje colaborativo

\begin{tabular}{|c|c|c|}
\hline $\begin{array}{l}\text { Estrategias } \\
\text { didácticas }\end{array}$ & Descripción de actividades ylo uso de materiales & $\begin{array}{c}\text { Documentado } \\
\text { por }\end{array}$ \\
\hline $\begin{array}{l}\text { Organizar tutorías } \\
\text { entre estudiantes }\end{array}$ & $\begin{array}{l}\text { Se definen las fortalezas y debilidades que tienen los niños y niñas para } \\
\text { que identifiquen que temas podrían explicar a otros compañeros, así como } \\
\text { los contenidos en los que requieren apoyo. }\end{array}$ & \multirow[t]{2}{*}{ Cedillo (2004) } \\
\hline $\begin{array}{l}\text { Trabajo por } \\
\text { Proyectos }\end{array}$ & $\begin{array}{l}\text { Partiendo del juego y la resolución de problemas los y las estudiantes } \\
\text { construyen aprendizajes significativos mientras se favorece la participación } \\
\text { activa y cooperativa en el aprendizaje. }\end{array}$ & \\
\hline $\begin{array}{l}\text { Emplear } \\
\text { juegos para la } \\
\text { interacción entre } \\
\text { estudiantes }\end{array}$ & $\begin{array}{l}\text { Se favorece el encuentro e interacción entre estudiantes a partir del uso de } \\
\text { juegos de mesa, saltar la soga, juegos de astucia y estrategia, el cine y los } \\
\text { títeres entre otros. }\end{array}$ & Jaramillo (2019) \\
\hline $\begin{array}{l}\text { Crear dibujos } \\
\text { para recuperar } \\
\text { conocimientos } \\
\text { previos de es- } \\
\text { tudiantes }\end{array}$ & $\begin{array}{l}\text { A partir de la creación de dibujos por parte de los niños y niñas es posible } \\
\text { recuperar conocimientos valiosos que quizá no están dentro del currículum } \\
\text { oficial, pero enriquecen la clase, como sus saberes en cuanto al cultivo de } \\
\text { maíz y las condiciones migratorias de sus familiares. }\end{array}$ & Gutiérrez (2013) \\
\hline $\begin{array}{l}\text { Emplear recursos } \\
\text { lúdicos para fa- } \\
\text { cilitar la comuni- } \\
\text { cación }\end{array}$ & $\begin{array}{l}\text { Ante la complejidad de atender grupos donde convergen una gran varie- } \\
\text { dad de idiomas, el uso de dibujos, mímica y otros recursos lúdicos podrían } \\
\text { beneficiar la comunicación. }\end{array}$ & $\begin{array}{l}\text { Vera y Yocupicio } \\
(2007)\end{array}$ \\
\hline $\begin{array}{l}\text { Creación de } \\
\text { talleres de } \\
\text { formación so- } \\
\text { ciomoral para la } \\
\text { comunidad. }\end{array}$ & $\begin{array}{l}\text { Consiste en una serie de actividades que lleven a las personas de la comu- } \\
\text { nidad hacia una acción a favor del género humano, y del reforzamiento del } \\
\text { tejido social. Utilizar los recursos limitados y que sean las mismas familias } \\
\text { migrantes quienes repartan pensando en quienes menos tienen. El ejerci- } \\
\text { cio es interesante y se recomienda se consulte el artículo original. }\end{array}$ & Hirsh (2009) \\
\hline
\end{tabular}

Fuente. Elaboración propia con base en Cedillo (2004), Jaramillo (2019), Gutiérrez (2013) y Vera y Yocupicio (2007).

Por último, Cedillo (2004) propone el empleo de tutorías y trabajo por proyectos como estrategias importantes para el desarrollo del trabajo colaborativo. Además, a través del juego es posible desarrollar 
actitudes de colaboración que permitan el encuentro intercultural, la resolución de conflictos y la participación activa de los y las estudiantes (Jaramillo, 2019; Cedillo, 2004).

Interculturalidad

Una parte importante de los NNAJAM son indígenas, por lo cual a continuación se reseñan las estrategias para educar para la interculturalidad en las escuelas que atienden a hijos de jornaleros. En primer lugar, Zambrano y Ávila (2018, pg. 353) consideran central que el docente realice un "análisis crítico de la realidad de las relaciones subordinadas que guardan las familias migrantes en su región de origen y en los lugares de llegada" y colocar la reflexión de la historicidad de la desigualdad de los jornaleros agrícolas como uno de los ejes de trabajo escolar pues la comprensión de los patrones de la interacción en el aula pretende proporcionar puntos de referencia para el diseño de innovaciones que procuren combatir prejuicios basados en estereotipos sobre grupos sociales diferenciados culturalmente.

Segura y Hernández (2018), documentaron en un centro escolar de Chihuahua la existencia de interacciones discriminatorias (de rechazo étnico y burlas hacia quienes no hablan español) entre alumnos indígenas y mestizos, incluso entre estudiantes de diversas etnias. Por lo cual, Chapela (2008, p. 25) sugiere "para relacionarnos con respeto, desde nuestros propios puntos de vista, conocimientos y valores, con otra persona que tiene sus propios puntos de vista, conocimientos y valores, las narraciones y las descripciones resultan indispensables". Se proponen las siguientes estrategias para trabajar con NNAJAM: entrevistas, debates, juegos, construcciones colectivas y creación de poemas.

\section{Alimentación y nutrición}

Para la enseñanza para una alimentación saludable (Melgar y Hernández, 2019) se considera necesaria pues a partir de una educación que tenga presente estos tópicos, puede beneficiar a la salud de los migrantes, a mejorar las acciones dirigidas y fortalecer estrategias educativas (Cuesta y Skolnik, 2013). Si bien es cierto que los programas de índole educativos sean centran en la adquisición de aprendizajes en la atención en temas como lectura, escritura y matemáticas, el aspecto de la alimentación es puesto en segundo orden en ocasiones.

La propuesta es desarrollar un currículo que tenga la dimensión alimenticia presente porque los padres y madres no tienen información al respecto. Este currículo desarrollaría temas como las proteínas y nutrimentos en los grados superiores de primaria, y serían los docentes los encargados de desarrollar estrategias para intervenir y poder incidir de forma positiva en problemas de sobrepeso, nutrición y alimentación (Melgar y Hernández, 2019). En caso de que se considerara esta opción, también los autores establecen que los docentes en su gran mayoría (más del 90\%) desconoce el tema por lo que habría que capacitarlos para dominar el tema de nutrición y que puedan desarrollarlo en la clase y que los alumnos lo apliquen en sus realidades cotidianas.

\section{El uso del tiempo libre}

Jaramillo (2019) hace una revisión respecto al uso del tiempo libre para el aprendizaje comparando diferentes modelos educativos elegidos. La autora para la educación formal modelo Montessori mientras que para la informal utiliza la pedagogía Scout Interamericana ${ }^{6}$ y a través de ellas, desarrolla propuestas educativas generales que aprovechan el tiempo libre que tienen los estudiantes de un campamento jornalero agrícola ubicado en Morelos. Resulta interesante ver que surgen de la revisión teórica y de un diálogo con profesores y estudiantes. Los primeros indicaban las actividades que bajo su percepción interesaban más a los estudiantes, a través de actividades ordenaban sus propios intereses (Jaramillo, 2019).

Una premisa importante es entender que el tiempo libre también se puede utilizar para detonar aprendizaje de los NNAJAM y que el juego y las actividades lúdicas son un mediador efectivo. La autora propone tres ejes principales para dividir las actividades y el tiempo libre en:

6 Según la misma autora, el método Scout tiene como objetivo educativo principal es formar ciudadanos sanos, dotados de conocimiento, de carácter y salud mientras que el método Montessori busca que el estudiante aprenda a pensar y actuar por sí mismo. Ambos evitan la competencia y buscan el trabajo colaborativo por lo que pueden ser comparados. 
1. Eje artístico: Propone actividades como danza, canto, pintura, música y teatro junto con otras manualidades. Esto permitiría el desarrollo de la personalidad de los niños y niñas aportando en la creatividad, autoconfianza y aceptación.

2. Eje de recreación: Orientado a la parte socioafectiva y coadyuva a mitigar problemas como depresión, consumo de drogas, entre otros. Para ello se requieren juegos de mesa, juegos de estrategia y títeres. Todo ello en teoría abre caminos hacia una vida más satisfactoria.

3. Eje deportivo: para detonar beneficios en el desarrollo físico y corporal principalmente la fuerza, resistencia, destreza y habilidad. Este eje no se aislado ya que también fortalece el trabajo en equipo, competitividad y responsabilidad.

Esta planeación debe ser propuesta por un "animador" (símil de la figura docente) y se encargaría de la logística de todas las actividades, lo que representa también tener que crear una figura administrativa que hasta ahora no está contemplada.

\section{Reflexiones finales}

Para fortalecer los procesos de aprendizaje de temas escolares de niños, niñas y adolescentes jornaleros agrícolas migrantes es deseable que las figuras educativas aprovechen, como recursos pedagógicos, a los contextos sociales, culturales, económicos y medioambientales en el que se desenvuelven los estudiantes, tanto en sus lugares de origen como en las regiones agrícolas donde radican de forma temporal.

Los procesos migratorios experimentados por los NNAJAM, les permiten contar con elementos contextuales de diversas regiones del país, lo cual es poco común en el alumnado que radica en las zonas rurales. Por lo cual, la enorme diversidad de experiencias, conocimientos y saberes constituyen elementos que caracterizan a los estudiantes NNAJAM, aspectos que podrían ser considerados como fortalezas con enormes potencialidades pedagógicas por parte de los docentes.

Las formas de alimentarse, el juego, el uso del tiempo libre, la repartición de bienes de consumo entre familias, por mencionar algunos, son elementos que permiten relacionar los temas escolares en la vida cotidiana y comunitaria, dándoles mayor sentido y convirtiéndose en aprendizajes significativos.

Sobre las estrategias didácticas, identificamos que, a pesar de los limitados programas y acciones educativas diseñadas por las autoridades de forma específica para los NNAJAM, las figuras educativas han desarrollado diferentes estrategias de enseñanza que procuran ser pertinentes al contexto y significativas. Sin embargo, la falta de documentación de éstas impide compartir aquellas que son exitosas, así como los retos que se enfrentan y consejos para ser implementadas.

En ese sentido, sería deseable promover espacios presenciales y virtuales que faciliten el intercambio de experiencias pedagógicas entre las figuras educativas que atienden escuelas rurales generales, multigrado, indígenas y las que trabajan con NNAJAM. Finalmente, las escuelas y poblaciones comparten características comunes que bien pueden permitir que el conocimiento, contextualización e implementación de estrategias enriquezcan el trabajo áulico y comunitario que desarrollan los docentes.

La invisibilidad de este sector es preocupante. La documentación e investigación que se tiene de estos grupos es muy poca lo que dificulta poder mantener información actualizada y proyectos efectivos a las necesidades y realidades de los NNAJAM. Como consecuencia, prevalece un estado de vulnerabilidad y desventaja de los jóvenes frente al contexto en el que se desenvuelven. Por otro lado, sigue siendo un reto que todas las estrategias tengan una visión intercultural, rural y que dispongan de los recursos adecuados.

\section{Referencias}

Arteaga, P. (2009). Los saberes docentes de profesores en escuelas con grupos multigrado [Tesis de Maestría]. México: CINVESTAV. 
Ávila, L. (2013). Pertinencia de técnicas de enseñanza de segundas lenguas en clases de matemáticas en contextos multilingües [Ponencia]. I Congreso de Educación Matemática de América Central y el Caribe. Santo Domingo, República Dominicana.

Ávila, A. (2014). La etnomatemática en la educación indígena: así se concibe, así se pone en práctica. Revista Latinoamericana de Etnomatemática, 7(1), 19-49. https://bit.ly/2XGO1f0.

Blancas, E. (2017). La alfabetización en español: los niños indígenas en la escuela general [ponencia]. XIV Congreso Nacional de Investigación Educativa. San Luis Potosí, México.

Cedillo, S. (2004) Educación primaria intercultural. Población infantil de hijos jornaleros agrícolas migrantes [Tesis no publicada de Licenciatura en Pedagogía]. México: UNAM.

Coordinación General de Educación Intercultural y Bilingüe- CGEIB (2002). Fomentar y mejorar la educación intercultural para los migrantes, proyecto presentado al Fideicomiso Fondo de Cooperación Técnica y Científica México-España, CGEIB-SEP.

Consejo Nacional de Fomento Educativo- CONAFE (1999). Educación Intercultural. Una propuesta para población infantil migrante. CONAFE.

Cuesta, G. y Skolnik, K. (2013). Un programa para la primera infancia adaptado a los hijos de los trabajadores migrantes y temporeros del campo (págs. 8-12). En Fundación Bernard van Leer, Espacios para la infancia. Fundación Bernard van Leer. https://bit.ly/38O1Ka9

Chapela, L. (2006). Percute tu atabal. México: Coordinación General de Educación Intercultural y Bilingüe.

Díaz Barriga, F. y Hernández Rojas, G. (2010). Estrategias Docentes para un aprendizaje significativo. Una interpretación constructiva. McGraw Hill.

Diario Oficial de la Federación- DOF (2014, 27 de diciembre). Acuerdo número 24/12/14 por el que se emiten las Reglas de Operación del Programa para la Inclusión y la Equidad Educativa para el ejercicio fiscal 2015. México.

Feltes, J. y Reese L. (2014). La implementación de programas de doble inmersión en escuelas multigrados rurales indígenas. Sinéctica. Revista Electrónica de Educación, (43), 01-18. https://bit. ly/3qnngZo

Ferreiro, R. (2005). Estrategias didácticas del aprendizaje cooperativo: el constructivismo social; una nueva forma de enseñar y aprender. Trillas

García, E. (2017). Enseñanza de las matemáticas en escuelas para migrantes [Ponencia]. XIV Congreso Nacional de Investigación Educativa. San Luis Potosí, México. https://bit.ly/2LQCAPg

Garduño, T. (2000, julio). Modelo educativo de comprensión intercultural. Consejo Nacional de Fomento Educativo [Ponencia]. III Conferencia de Investigación Socio-Cultural. Campinas, Brasil. Disponible en https://bit.ly/35MOj8B

Galván, L. y Espinosa, L. (2017). Diversidad y prioridades educativas en escuelas multigrado. Estudio de caso en México. Sinéctica. Revista Electrónica de Educación (49), 1-19. https://bit.ly/35KvCma

Glockner, V. (2019). Los estudios sobre infancia jornalera en México: aportaciones, retos y futuras posibilidades para la expansión del campo de estudio . Textual, 74, 391-720. https://bit.ly/3qne9la

Gutiérrez, N. (2013). Producción y circulación de conocimiento en comunidades agrícolas. Prácticas productivas y aprendizaje en la vida cotidiana. CPU-e, Revista de Investigación Educativa, (17), 128-150. https://bit.ly/35IZ37N

Hirsch, A. (2010). Aprender a aprender y a convivir. Fundamentos teóricos de una estrategia educativa para familias jornaleras migrantes. Perfiles Educativos, 32(127), 152-156. https://bit.ly/35JJj4F

Grammont, H. y Martínez, L. (2009). La pluriactividad en el campo latinoamericano. FLACSO Ecuador. https://bit.ly/2MXSaJw 
Instituto Nacional de Evaluación de la Educación (INEE) (2018). Evaluación de las intervenciones públicas y programas de escuelas multigrado. Reporte de Evaluación. https://bit.ly/2XNLv6A

Instituto Nacional de Evaluación de la Educación (INEE) (2016). Directrices para mejorar la atención educativa de niñas, niños y adolescentes de familias de jornaleros agrícolas migrantes. https:/l bit.ly/3igdANz

Instituto Nacional de Evaluación de la Educación (INEE) (2019). Panorama educativo estatal de la población indígena 2018. UNICEF-INEE. https://bit.ly/3skTJRQ

Jaramillo, C. (2019). Atención a los intereses lúdicos y de aprovechamiento del tiempo libre para hijos/as de jornaleros agrícolas migrantes [Tesis de Maestría]. Morelos, México: Universidad Autónoma del Estado de Morelos.

Juárez, D. y Lara, E. (2018). Procesos de enseñanza en escuelas rurales multigrado de México mediante Comunidades de Aprendizaje. Tendencias Pedagógicas, (31), pp. 149-163. http://dx.doi. org/10.15366/tp2018.31.009

Melgar, M., y Hernández, E. (2019). La enseñanza para una alimentación saludable en escuelas para la niñez migrante -un estudio exploratorio-. Revista Latinoamericana de Educación y Estudios Interculturales, 3, 57-68.

Méndez, A. y Huguette, P. (2013). Aprendizajes en el surco y en la escuela en niños y niñas de familias jornaleras agrícolas migrantes en Michoacán [Ponencia]. XII Congreso Nacional de Investigación Educativa. Guanajuato, México. https://bit.ly/2KISgK1

Montoya, L. y Villareal, A. (s/f). Estrategias para favorecer la lecto-escritura en niños migrantes de primer grado de primaria. [Proyecto de intervención pedagógica]. México: Universidad Pedagógica Nacional Unidad 25-A. https://bit.ly/3nOHmKj

Pacheco, L., Cayeros, L. y Madera, J. (2016). Interculturalidad y derecho a la educación de la niñez indígena jornalera migrante. LiminaR, 14 (1), 92-105.

Ramírez, M. (2000). Situación de vulnerabilidad de las niñas y niños migrantes en México. Problemática para su acceso a una educación de calidad. En Norma del Río (Coord.), La infancia vulnerable de México en un mundo globalizado. (Págs. 55-78). México: UAM / UNICEF.

Ramírez, A. (2008). Diseño didáctico con enfoque colaborativo para escuelas de niños migrantes en Sonora [Tesis no publicada de Maestría en Innovación Educativa]. Hermosillo, México: Universidad de Sonora.

Rojas, T. (2006). Resultados de una política orientada hacia la equidad y calidad de la educación primaria para las niñas y niños jornaleros migrantes. Revista Estudios Sociales, 14(27), 94-122.

Rojas, T. (2011). Inequidades: la educación primaria de niñas y niños jornaleros agrícolas migrantes. México: Universidad Pedagógica Nacional.

Rubio, R. (2009). El programa de lectura para niñas y niños migrantes de educación primaria. [Tesis de Licenciatura]. México: Universidad Pedagógica Nacional.

Schmelkes, S. (2002). La enseñanza de la lectura y la escritura en contextos multiculturales [Ponencia]. VII Congreso latinoamericano para el desarrollo de la lectura y la escritura. Puebla, México.

Secretaría de Educación Pública (SEP). (2006). Guía para el docente Español y el medio natural y social. Primer Ciclo. México: PRONIM/ SEP.

Secretaría de Educación Pública (SEP). (2019). Programa para la Inclusión y la Equidad Educativa 2017 - 2018 (Consistencia y Resultados). Formato Aspectos Relevantes de la Evaluación. México. https://bit.ly/3su36P8

Secretaría de Desarrollo Social (SEDESOL) (s/f). Programa de Atención a Jornaleros Agrícolas. [Página oficial]. https://bit.ly/3irAQby 
Secretaría de Desarrollo Social (SEDESOL) (2011). Pobreza, migración y capacidades básicas en la población jornalera agrícola en México. Resultados de la encuesta nacional de jornaleros https://bit.ly/3qoY4lg. México: SEDESOL.

Segura, T. y Hernández, O. (2018). Niñez inmigrante e interculturalidad escolar en el norte de Chihuahua. Región y Sociedad, 30 (73). https://bit.ly/3qoY4lg

Solares, D. (2012a). Conocimientos matemáticos en situaciones extraescolares. Análisis de un caso en el contexto de los niños y niñas jornaleros migrantes. Educación Matemática, (24), 1, 5-33. https://bit.ly/3igmk6n

Solares-Pineda, D. V. (2012b). Conocimientos matemáticos de niños y niñas jornaleros migrantes: algunas preguntas para la escuela. Rayuela. Revista Iberoamericana sobre Niñez y Juventud en la Lucha por sus Derechos. 2(4), 101-110. https://bit.ly/3ss6YjH

Solares, D. y Block, D. (2017). “¿Dónde conviene cambiar el cheque?” Conocimientos multiplicativos en alumnos jornaleros agrícolas migrantes, Sinéctica, 49, 1-18. https://bit.ly/3bl4hET

Salinas, S. (2004). Educación intercultural con jornaleros migrantes: conceptos y estrategias [ponencia]. Cuartas jornadas sobre infancia. Redes de formación e investigación para la promoción de los derechos de la infancia. Ciudad de México, 21 de septiembre a 23 de noviembre. https://bit. ly/3qkdUxn.

Vera, J. y Yocupicio, J. (2007). Aprendizaje colaborativo en aulas multigrado en escuelas de niños migrantes. En J. Homero (coord), Mentes híbridas. Lo que la migración y la educación hacen en contexto. (Pp. 135-160). México: UPN - CONACYT.

Zambrano, A. y Ávila, L. (2018). Sociabilidad restringida en aulas multiculturales con niños jornaleros itinerantes. Revista Mexicana de Investigación Educativa, 23(77), 351-379. https://bit.ly/3ihEnsV 